\title{
The reminiscence bump for salient personal memories: Is a cultural life script required?
}

\author{
Ryan A. Dickson - David B. Pillemer • \\ Elizabeth C. Bruehl
}

Published online: 22 March 2011

(C) Psychonomic Society, Inc. 2011

\begin{abstract}
Older adults report a higher frequency of autobiographical memories for experiences that occurred between ages 15 and 30, as compared with other life periods. This reminiscence bump is evident for memories involving positive, but not negative, emotions. The cultural life script hypothesis proposes that people share knowledge for the types and timing of positive landmark events and that this script guides the memory search to the bump period. The present research examined whether a reminiscence bump would be evident when memory cues prompted recall of surprising and unexpected events. Older adults recalled positive and negative, surprising positive and surprising negative, or highly expected and highly unexpected events. Adults' memory distributions were compared with distributions of predicted life events generated by undergraduates. Reminiscence bumps were found not only for memories of positive and expected events, but also for memories of surprising and unexpected events. Implications for the life script account are discussed.
\end{abstract}

Keywords Autobiographical memory $\cdot$ Recall $\cdot$ Emotion

Researchers examining the distribution of autobiographical memories over the life span have consistently found that people over age 40 report a higher frequency of memories for experiences that occurred during adolescence and young adulthood than for any other life period (Berntsen \& Rubin, 2004; Rubin \& Berntsen, 2003; Rubin, Wetzler, \& Nebes, 1986). This reminiscence bump has been found across cultures (Conway, Wang, Hanyu, \& Haque, 2005; Janssen,

R. A. Dickson • D. B. Pillemer $(\bowtie) \cdot$ E. C. Bruehl

Department of Psychology, University of New Hampshire, Durham, NH 03824, USA

e-mail: david.pillemer@unh.edu
Chessa, \& Murre, 2005) and through the use of a variety of methods, including responses to word cues (Jansari \& Parkin, 1996; Rubin \& Schulkind, 1997), olfactory cues (Chu \& Downes, 2000), and musical cues (Schulkind \& Woldorf, 2005); it has been found for participants' most vivid (Fitzgerald, 1988; Robinson \& Taylor, 1998; Webster \& Gould, 2007) and most important (Berntsen \& Rubin, 2002) autobiographical memories, as well as for life chapters (Thomsen \& Berntsen, 2008) and stories that belong in a book about one's life (Fitzgerald, 1996).

The reminiscence bump is a robust finding within the autobiographical memory literature, but it has not been found for all classes of memories. When adults are asked to think back over their lives and identify salient emotional or important events, a bump is evident in the distribution of positive, but not negative, memories (for an exception, see Davison \& Feeney, 2008, for memories of regret). Rubin and Berntsen (2003; Berntsen \& Rubin, 2002, 2004) have argued that this divergent pattern of findings for positive and negative memories is best explained by a cultural life script hypothesis: "The retrieval of autobiographical memories is governed by culturally shared representations of the prototypical life cycle that locate the majority of important transitional events in young adulthood and favor positive events" (p. 2). Examples of positive transitional milestones include marriage and childbirth. In contrast, because negative events are often unanticipated (e.g., a car accident) or, when expected, are less temporally restricted (e.g., the death of a parent), they do not become part of a culturally shared life script and are not overrepresented in any particular life period.

Several recent studies have provided empirical support for the existence of a cultural life script. For example, Rubin and Berntsen (2003) asked Danish adults to provide the age at which they had their most important experience, when they had felt most in love, most proud, most afraid, 
most angry, and most jealous. The researchers then asked undergraduate students to imagine an average 70 -year-old who is reflecting back over his or her life and to estimate the person's age when he or she likely experienced these emotional events. The distributions of predicted and actual positive memories were highly similar and demonstrated reminiscence bumps. In contrast, predicted and actual negative memory distributions did not show increases in frequency during adolescence and young adulthood. These results have been largely replicated across cultures (Erdoğan, Baran, Avlar, Çağlar Taş, \& Tekcan, 2008; Habermas, 2007; Rubin, Berntsen, \& Hutson, 2009) and among people of varying ages (Bohn, 2009; Bohn \& Berntsen, 2008; Janssen \& Rubin, 2011).

Other theories developed to account for the bump (for a review, see Berntsen \& Rubin, 2002) include a cognitive perspective (Rubin, Rahhal, \& Poon, 1998; Schrauf \& Rubin, 1998, 2001), a biological or maturational perspective (Janssen, Murre, \& Meeter, 2008), and a narrative (Elnick, Margrett, Fitzgerald, \& Labouvie-Vief, 1999; Fitzgerald, 1988, 1996, 1999), life story (Bluck \& Habermas, 2000, 2001; Glück \& Bluck, 2007), or identity (Conway, 2005; Conway \& Haque, 1999; Conway \& Holmes, 2004; Holmes \& Conway, 1999) perspective. Because these alternative accounts do not predict differences in the distributions of positive and negative memories, they cannot readily explain the overrepresentation of positive memories in the bump period (Berntsen \& Rubin, 2002) and are not a primary focus of this research.

A final explanation for the divergent distributions of positive and negative memories across the life span is that they reflect the actual distributions of subjectively experienced positive and negative events. Evidence supporting a subjective reality perspective has come primarily from generational differences in autobiographical memory. For example, Berntsen and Rubin (2002) and Rubin and Berntsen (2003) asked a representative sample of Danes to report their age when they had experienced a number of emotional events, including memories of fear and trauma. For participants 70 to 94 years of age, these negative memories were overrepresented in late adolescence and early adulthood, a time period corresponding to the German occupation of Denmark. The distributions of fear and trauma memories from preinvasion through postinvasion conform to what one would actually experience as an antagonistic foreign military takes up offensive positions and readies for invasion (fear), invades (trauma in addition to fear), occupies (modest decrease or leveling off of trauma and fear), and finally ends occupation (sharp decreases in both trauma and fear). Rubin and Berntsen argued that these findings reflect a cohort effect in which adults who experienced events related to World War II developed a specific "war generation" life script (p. 7).
Alternatively, memories could reflect the actual distribution of subjectively experienced emotional memories, kept alive by their unique and dramatic content, rather than by their resonance with a cohort-specific, higher order life script. As sung by Roger Waters of Pink Floyd, it may simply be that "the memories of a man in his old age are the deeds of a man in his prime" (1972).

In summary, the life script account provides the most convincing explanation for the robust difference between the temporal distributions of positive and negative memories. Yet it remains unclear whether the theoretical construct of a life script is required - whether life scripts do in fact "structure recall from autobiographical memory" (Berntsen \& Rubin, 2004, p. 427). The overrepresentation of positive memories in the bump period could be consistent with a life script, and yet the script may not always play a critical role in organizing autobiographical memory and directing the memory search.

\section{The present research}

To determine whether the reminiscence bump depends on the activation and directive influence of a life script, we manipulated one of the life script's defining properties, expectedness (Berntsen \& Rubin, 2004). We examined temporal distributions of memories provided in response to prompts for surprising or unexpected events, as well as standard or expected events. In addition, an examination of memory content is necessary to determine whether memories of scripted events are overrepresented in the bump period, as compared with the nonbump period. While Berntsen and Rubin (2002, 2004; Rubin \& Berntsen, 2003) did not have respondents give detailed memory descriptions, several recent studies have confirmed that scripted positive events frequently populate the bump period (Bohn, 2009; Collins, Pillemer, Ivcevic, \& Gooze, 2007; Thomsen \& Berntsen, 2008). In the present studies, content analyses of surprising and unexpected predicted events and memories were used to clarify the role played by a cultural life script in producing the reminiscence bump.

In four studies, we examined the content and age distributions of college student predicted events and older adult memories. In Study 1, college students were asked to imagine a hypothetical 70-year-old and to describe either an especially positive and an especially negative event or a surprising positive and a surprising negative event that the person would have experienced from any point across the life span. College student predictions provided an assessment of shared cultural knowledge concerning the probable timing and content of standard and surprising life events. In Study 2, older adults provided detailed accounts of their own memories in response to these prompts. In Studies 3 
and 4 , the emotional cue (i.e., positive or negative) was eliminated, and the role played by expectedness was examined directly. In Study 3, college students predicted highly expected and highly unexpected events from across the life span, and in Study 4, older adults recalled memories of highly expected and highly unexpected events. Only after providing memory descriptions were participants in Studies 3 and 4 asked to rate both the positive and negative valence of each memory.

\section{Hypotheses: Studies 1 and 2}

We expected that the age distributions of especially positive and negative predicted events (Study 1) and older adult memories (Study 2) would replicate the results of prior studies. Positive, but not negative, predicted events and actual memories should show reminiscence bumps between ages 16 and 30. In addition, content analyses should reveal that positive events and memories in the bump period frequently reference life scripts. Several outcomes are possible regarding surprising positive and negative events and memories. For simplicity, these outcomes are described for adult memories (Study 2) but also apply to college student predicted events (Study 1).

Berntsen and Rubin (2004) proposed that some memory tasks-such as a request for positive and important memories - are especially likely to activate the life script, which then guides memory retrieval. Because the cultural life script is composed only of "important, expected transitional events" (Berntsen \& Rubin, 2004, p. 429), asking for memories of surprising events may lessen the likelihood that a life script will be activated. If the reminiscence bump is dependent on the activation of a life script, both surprising positive and negative memory distributions will be relatively flat, and memory content should rarely reference cultural landmarks.

Alternatively, the surprising negative memory prompt could first activate the life script and then trigger a search for expected positive outcomes that were not realized. If surprising negative memories represent failures to achieve expected cultural landmarks (e.g., having a college application or marriage proposal rejected), the distribution of these memories may show an increased frequency in late adolescence and early adulthood. The surprise cue would reverse the usual effects of emotional valence such that the bump is apparent for negative, but not positive, memories. This reverse pattern of results would nevertheless be consistent with the hypothesized guiding influence of life scripts.

Berntsen and Rubin (2004) proposed that life scripts "provide search descriptions for times when one is most likely to have experienced the emotion concerned" (p. 430).
Accordingly, a request for a surprising positive memory may activate the life script, which then guides the memory search to the age period most likely to be populated by positive landmark events, from which an individual then searches for a memory that has an aspect of surprise. In this case, the distribution of surprising positive (but not negative) memories will show a reminiscence bump. Content analyses of bump period memories should reveal a high frequency of landmark events, but with a surprising element.

\section{Study 1}

Similar to the methods used in previous research (e.g., Berntsen \& Rubin, 2004), we examined cultural scripts for emotionally salient events by asking college students to imagine a hypothetical older adult thinking about his or her life and to describe and date events that the person would have experienced.

\section{Method}

Questionnaire College students were asked to imagine an average or typical 70-year-old (not someone they knew) of their same gender who is looking back over his or her life, thinking about a range of different events. Participants were then asked to describe specific events that either were especially positive and especially negative or were surprising in an especially positive way and surprising in an especially negative way that the hypothetical 70 -year-old would have experienced (counterbalanced within expectedness condition). Participants were asked to estimate how old the 70-year-old was when he or she experienced the event, as well as their confidence that the age estimate they provided fell within a decade of the actual experience and the extent to which they experienced difficulty thinking of the event (the latter questions were included for exploratory reasons and are not reported here). Across studies, for participants who reported an age range, an average age was calculated. Participants completed questionnaires in a group setting. The four questionnaire versions were passed out to participants in alternating order.

Participants Of the 202 participants who completed the questionnaire, 4 were dropped for failure to follow instructions $(n=198)$. The majority of participants $(92.4 \%)$ selfidentified as European, Caucasian, or White, females made up $82.8 \%(n=164)$ of the sample, and the mean age of participants was 18.67 years $(S D=1.76$; range $=17-33)$. Although female participants are overrepresented in the sample, previous studies have failed to show major effects of gender on life scripts (e.g., Erdoğan et al., 2008). 
Content coding The first and third authors read all event descriptions, created content categories reflecting major themes for predicted positive and negative events (collapsed across expectedness), and coded all event descriptions. The content analysis was open-ended and was not restricted to categories identified in previous studies. Categories reaching 3.0\% were retained-all other events were folded into an "other" category (similar to the procedure used by Bohn, 2009) — and differences were resolved through discussion. A research assistant, blind to hypotheses, coded a random sample of $30.0 \%$ of the event descriptions, resulting in $96.67 \%$ agreement $(\kappa=.96)$ for predicted positive events and $93.33 \%$ agreement $(\kappa=.92)$ for predicted negative events.

\section{Results}

To examine temporal distributions, predicted events were divided into 15-year age bins $(0-15,16-30,31-45$, and 4660; figures display an additional 61 years and older age bin). The 16- to 30-year age bin represents the predicted location of the reminiscence bump (e.g., Berntsen \& Rubin, 2004). To enable comparisons with Studies 2, 3, and 4, statistical tests for all studies include the four age bins dated prior to age 61 (all Study 2 participants were at least 60 years old, and analysis of Study 4 data focused on participants 60 years and older).

Distributions of predicted events are presented in Fig. 1 (for all figures, error bars represent standard errors). As was predicted, especially positive events produced a pronounced bump between ages 16 and 30, with $80.20 \%$ of the events falling within this age interval, $\chi^{2}(3)=168.08, p<.001$. Surprising positive events also produced a reminiscence bump, with $58.42 \%$ of the predicted events falling between ages 16 and $30, \chi^{2}(3)=80.21, p<.001$. A more modest

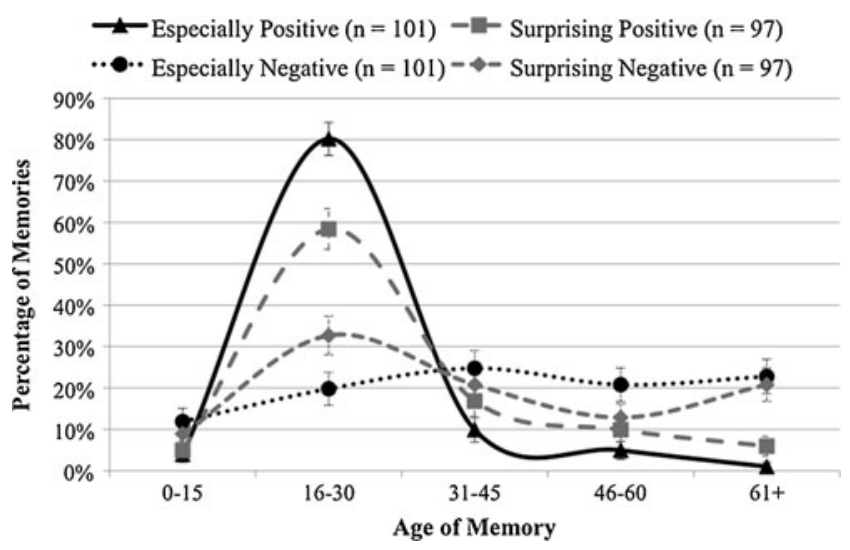

Fig. 1 Age distributions of positive, negative, surprising positive, and surprising negative events predicted by college students (Study 1) bump is evident for surprising negative events, with $32.67 \%$ of predicted events falling between ages 16 and $30, \chi^{2}(3)=$ $17.68, p=.001$, whereas the distribution of especially negative events is relatively flat, $\chi^{2}(3)=4.56, p=.207$.

Table 1 (especially positive and surprising positive events) and Table 2 (especially negative and surprising negative events) present content categories by bump period. The majority of especially positive events in the bump period focused primarily on two major transitions or landmarks occurring in early adulthood (marriage and having a child), whereas surprising positive bump period events prominently included surprising subevents of these major landmarks (proposal or engagement, learning of pregnancy, and meeting significant other). A comparison of surprising negative bump period and nonbump period events reveals that two content categories-war and the Pearl Harbor attack-are clearly overrepresented in the bump period.

\section{Discussion}

Similar to the findings reported in previous studies (Berntsen \& Rubin, 2004; Bohn, 2009), predicted positive events showed a sharp bump between ages 16 and 30 . Predicted surprising positive events also showed a pronounced bump. In contrast, the distribution of especially negative events was flat, and surprising negative events showed only a modest increase between ages 16 and 30 .

In general, predicted positive events were highly reflective of a cultural life script, in agreement with previous research. Especially positive events often corresponded to major life transitions (marriage and having a child), whereas surprising positive events often targeted these major transitions, but with an unexpected component (proposal or engagement and learning of pregnancy). These findings are consistent with the idea that a surprising positive prompt activates the life script, which then guides the memory search to the transitional age period heavily populated by positive scripted events, from which an individual then searches for an event containing an element of surprise.

\section{Study 2}

Older adults were asked to provide detailed accounts of their own especially positive and especially negative, or surprising positive and surprising negative, memories. According to the life script hypothesis, we should find an increase in the frequency of especially positive memories between ages 16 and 30, and positive bump period memories should often describe scripted events, including 
Table 1 Study 1 positive content categories by bump period and expectedness

\begin{tabular}{|c|c|c|c|c|}
\hline \multirow[t]{2}{*}{ Category } & \multicolumn{2}{|c|}{ Bump Period (16-30 Years) } & \multicolumn{2}{|c|}{ Nonbump Period } \\
\hline & Standard & Surprising & Standard & Surprising \\
\hline$n$ & 81 & 59 & 20 & 38 \\
\hline Other & $3.70 \%$ & $13.56 \%$ & $30.00 \%$ & $39.47 \%$ \\
\hline Marriage & $48.15 \%$ & $15.25 \%$ & $0.00 \%$ & $0.00 \%$ \\
\hline Having child & $30.86 \%$ & $11.86 \%$ & $20.00 \%$ & $0.00 \%$ \\
\hline College transition & $4.94 \%$ & $6.78 \%$ & $0.00 \%$ & $0.00 \%$ \\
\hline Career or finance advance & $4.94 \%$ & $5.08 \%$ & $20.00 \%$ & $21.05 \%$ \\
\hline Meeting significant other & $3.70 \%$ & $10.17 \%$ & $0.00 \%$ & $2.63 \%$ \\
\hline War resolution or reunion & $2.47 \%$ & $3.39 \%$ & $5.00 \%$ & $2.63 \%$ \\
\hline Proposal or engagement & $1.23 \%$ & $16.95 \%$ & $0.00 \%$ & $0.00 \%$ \\
\hline Learning of pregnancy & $0.00 \%$ & $16.95 \%$ & $0.00 \%$ & $7.89 \%$ \\
\hline Grandchild & $0.00 \%$ & $0.00 \%$ & $25.00 \%$ & $26.32 \%$ \\
\hline
\end{tabular}

events related to marriage and childbirth, as predicted by college students in Study 1. On the basis of students' predictions in Study 1, we also expected the distribution of surprising positive memories to peak in the bump period. In contrast, especially negative memories should not demonstrate a bump. In accordance with Study 1, the distribution of surprising negative memories should show, at most, a modest increase during the bump period.

\section{Method}

Questionnaire Study 2 memory prompts were identical to those used in Study 1, except that they referred to adults' own memories. Follow-up questions included participants' age at the time of the memory experience and the extent to which they were surprised by the original memory event (rated on a 1 to 5 scale; $1=$ not at all surprised, $5=$ extremely surprised). Other questions assessed discussion frequency, retrieval difficulty, and the emotional impact of the original event (these questions were included for exploratory reasons and are not reported here). For each memory, the adults in Studies 2 and 4 were asked to complete the short version of the Centrality of Events Scale (CES) developed by Berntsen and Rubin (2006). The CES was also included for exploratory reasons and is not discussed further.

Participants Older adults were recruited from a participant pool administered by the Cornell Institute for Translational Research on Aging (CITRA). This pool is composed of adults aged 60 years and older who live in Tompkins County, New York and have indicated a willingness to participate in research. At the time this research was conducted, the pool consisted of 842 adults, all of whom were contacted by mail for participation in this study. A recruitment letter described the present research and indicated its association with CITRA. Adults who agreed
Table 2 Study 1 negative content categories by bump period and expectedness

\begin{tabular}{|c|c|c|c|c|}
\hline \multirow[t]{2}{*}{ Category } & \multicolumn{2}{|c|}{ Bump Period (16-30 Years) } & \multicolumn{2}{|c|}{ Nonbump Period } \\
\hline & Standard & Surprising & Standard & Surprising \\
\hline$n$ & 20 & 33 & 81 & 64 \\
\hline Other & $15.00 \%$ & $15.15 \%$ & $3.70 \%$ & $7.81 \%$ \\
\hline Other death & $20.00 \%$ & $15.15 \%$ & $9.88 \%$ & $9.38 \%$ \\
\hline Parent death & $15.00 \%$ & $18.18 \%$ & $37.04 \%$ & $14.06 \%$ \\
\hline War & $15.00 \%$ & $12.12 \%$ & $6.17 \%$ & $1.56 \%$ \\
\hline Victimization or discrimination & $10.00 \%$ & $6.06 \%$ & $3.70 \%$ & $4.69 \%$ \\
\hline Pearl Harbor attack & $5.00 \%$ & $12.12 \%$ & $0.00 \%$ & $3.13 \%$ \\
\hline Accident or disease & $5.00 \%$ & $9.09 \%$ & $6.17 \%$ & $14.06 \%$ \\
\hline Career or finance difficulty & $5.00 \%$ & $6.06 \%$ & $4.94 \%$ & $7.81 \%$ \\
\hline Relationship difficulty & $5.00 \%$ & $6.06 \%$ & $3.70 \%$ & $10.94 \%$ \\
\hline Great Depression & $5.00 \%$ & $0.00 \%$ & $2.47 \%$ & $4.69 \%$ \\
\hline Spouse death & $0.00 \%$ & $0.00 \%$ & $22.22 \%$ & $21.88 \%$ \\
\hline
\end{tabular}


to participate were asked to complete the questionnaire and return it in a prepaid envelope. Compensation was provided in the form of a participant raffle for a $\$ 150$ gift certificate; raffle entry forms were returned separate from questionnaires. Reminder and debriefing letters were mailed to all participant pool members.

Of the 842 questionnaires mailed, 26 adults declined participation, and 12 questionnaires were returned due to incorrect address, were unopened, or were opened but not completed. Excluding these 38 adults, 281 questionnaires were returned at least partially completed (response rate $=$ $35.95 \%$ ); 22 participants were dropped for failure to follow instructions (e.g., reporting only one memory), resulting in a final sample of 259 participants. Almost all (97.7\%) participants self-identified as European, Caucasian, or White, females made up 59.5\% $(n=154)$ of the sample, and the mean age of participants was 70.65 years $(S D=$ 7.77 ; range $=60-93$ ).

Content coding Using the coding scheme generated from Study 1 college student predictions, the first and third authors coded all memories independently within valence, and differences were resolved through discussion. A research assistant, blind to the hypotheses, coded a random sample of $30.0 \%$ of the memories, resulting in $91.03 \%$ agreement $(\kappa=.85)$ for positive memories and $78.21 \%$ agreement $(\kappa=.73)$ for negative memories. Because the coding scheme generated from college student predictions captured only a fraction of adult positive memories $(52.51 \%$ coded "other"; $22.01 \%$ of negative memories coded "other"), the two researchers next coded memories using additional categories generated from actual adult memory content, as well as categories reported by Berntsen and Rubin (2004), and differences were again resolved through discussion. The research assistant then coded a random sample of $30.0 \%$ of memories initially coded "other," resulting in $73.17 \%$ agreement $(\kappa=.68)$ for positive memories and $64.71 \%$ agreement $(\kappa=.57)$ for negative memories. The higher intercoder agreement for students' predicted life events in Study 1 than for adults' memories in Study 2 may be attributable, in part, to differences in narrative complexity. Students' descriptions of predicted events tended to be short and clearly focused on a particular theme (such as marriage), whereas adults' descriptions of actual life events tended to be more extensive and multifaceted.

\section{Results}

To determine whether the surprise cue was effective, ratings of memories prompted by the standard and surprise cues were compared for positive and negative memories separately. Surprising positive memories $(M=3.99, S D=1.00)$ were perceived as more surprising than especially positive memories $(M=3.04, S D=1.37), t_{\text {adjusted }}(208.88)=6.20, p<.001$, $\eta^{2}=.16$, and surprising negative memories $(M=4.31, S D=$ $0.89)$ were perceived as more surprising than especially negative memories $(M=3.77, S D=1.32), t_{\text {adjusted }}(198.98)=$ 3.77, $p<.001, \eta^{2}=.07$.

Distributions of adult memories are presented in Fig. 2. As was expected, especially positive memories showed a pronounced bump, with $34.71 \%$ of events occurring between ages 16 and $30, \chi^{2}(3)=11.99, p=.007$. Surprising positive memories demonstrated an almost identical bump, with $34.78 \%$ of events occurring between ages 16 and $30, \chi^{2}(3)=16.56, p=.001 .^{1}$ In contrast, relatively flat distributions were evident for both especially negative, $\chi^{2}(3)=2.73, p=.436$, and surprising negative, $\chi^{2}(3)=1.30, p=.729$, memories.

Table 3 presents especially positive and surprising positive content categories by bump period. A comparison of especially positive bump period and nonbump period memories reveals that pregnancy or having a child and proposal or marriage are overrepresented in the bump period. For surprising positive memories, pregnancy or having a child and, to a lesser extent, college transition, meeting a significant other, and proposal or marriage are overrepresented in the bump period, as compared with the nonbump period.

\section{Discussion}

Similar to findings reported in previous studies, older adult positive memories showed a pronounced reminiscence bump between ages 16 and 30. A novel finding is that positive memories of surprising events produced a nearly identical bump. In support of the life script hypothesis, age distributions for both standard and surprise cues were generally consistent with predictions made by college students in Study 1, although student predictions exaggerated the size of the actual adult memory bumps.

Especially positive and surprising positive bump period memories frequently described well-known transitional events. These findings are consistent with one interpretation of the life script hypothesis, in which the request for a surprising positive memory directs the memory search to

\footnotetext{
${ }^{1}$ Although we had no a priori hypotheses concerning gender differences, exploratory analyses of adults' memories (Studies 2 and 4) were conducted. Distributions were similar for all memory probes, with two exceptions: The bumps for surprising positive memories in Study 2 and for unexpected positive memories in Study 4 were more prominent for males than for females.
} 


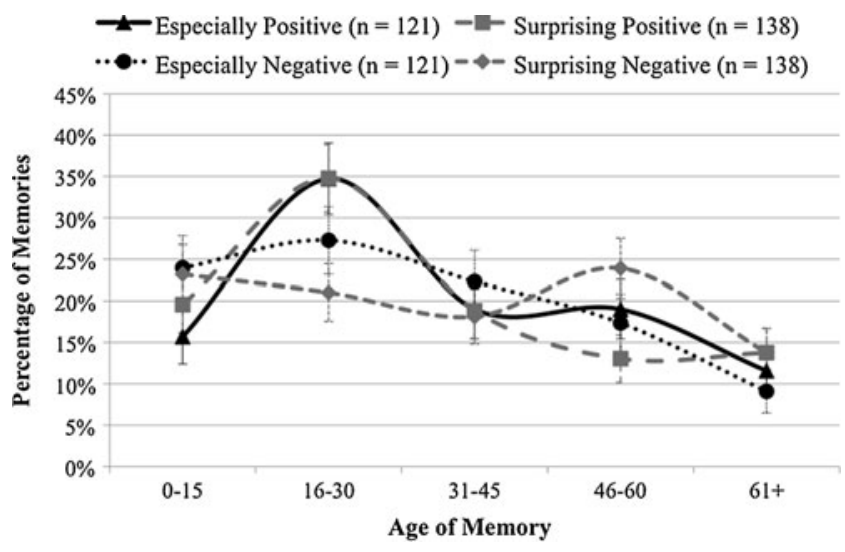

Fig. 2 Age distributions of positive, negative, surprising positive, and surprising negative memories for older adults (Study 2)

the life period most likely to contain positive events (Berntsen \& Rubin, 2004), from which an individual then searches for a memory that has an aspect of surprise.

\section{Overview of studies 3 and 4}

The conclusions based on Study 2 findings are limited by two methodological issues. First, the memory prompt "surprise" is not entirely neutral with regard to emotional valence. In Bradley and Lang's (1999) affective norms for English words, the term "surprised" was rated as moderately arousing and moderately positive. Talarico, LaBar, and Rubin (2004; Rubin \& Talarico, 2009) also found that memories generated in response to a "surprise" prompt were rated as moderately positive. If "surprise" is itself a positive term, then it may have worked in concert with the positive memory cue. In Studies 3 and 4, this issue was addressed by replacing "surprise" with memory prompts that more directly reflected an event's normative or scripted quality. College students were asked to predict, and older adults to recall, highly expected and highly unexpected events from across the life span.

A second issue concerns the distinctive roles played by expectedness and emotional valence in the memory search. In Studies 1 and 2, prompts manipulating expectedness (standard or surprising) and emotional valence (positive or negative) were presented in combination, such that we were unable to assess their independent contributions. In Studies 3 and 4 , we first requested a highly expected or highly unexpected memory and, after the memory had been described, participants rated its emotional valence. This allowed us to determine whether a reminiscence bump is apparent when memory probes target unexpected, as well as expected or scripted, events.

Hypotheses: Studies 3 and 4

Expected events In previous research (Berntsen \& Rubin, 2004; Bohn, 2009), the majority of landmark events predicted to occur over the course of one's life were considered positive. In addition, negative events are almost always unanticipated or, when anticipated (e.g., a parent's death), rarely occur within a narrow time span. Accordingly, most college student predictions and adult memories for highly expected events should be rated positive. Second, if a life script absent a corresponding positive cue is sufficient to drive the memory search to the period overrepresented by scripted events, we should find that student predictions and adult memories for highly expected events will be overrepresented between ages 16 and 30. Third, bump period expected events and memories rated positive should reflect cultural scripts to a large extent. Finally, a bump should not be evident for the
Table 3 Study 2 positive content categories by bump period and expectedness

Because the Study 1 predicted categories "proposal or engagement" and "learning of pregnancy" did not reach the $3.0 \%$ criteria, they were combined with the categories "marriage" and "having a child," respectively. All other predicted categories not reaching $3.0 \%$ were collapsed into the category "other"

\begin{tabular}{|c|c|c|c|c|}
\hline \multirow[t]{2}{*}{ Category } & \multicolumn{2}{|c|}{ Bump Period (16-30 Years) } & \multicolumn{2}{|c|}{ Nonbump Period } \\
\hline & Standard & Surprising & Standard & Surprising \\
\hline$n$ & 42 & 48 & 79 & 90 \\
\hline Other & $7.14 \%$ & $10.42 \%$ & $18.99 \%$ & $17.78 \%$ \\
\hline Pregnancy or having child & $21.43 \%$ & $20.83 \%$ & $3.80 \%$ & $5.56 \%$ \\
\hline Proposal or marriage & $19.05 \%$ & $6.25 \%$ & $1.27 \%$ & $0.00 \%$ \\
\hline Career or finance advance & $16.67 \%$ & $16.67 \%$ & $17.72 \%$ & $18.89 \%$ \\
\hline Meeting significant other & $11.90 \%$ & $12.50 \%$ & $7.59 \%$ & $5.56 \%$ \\
\hline Nonromantic relationship & $9.52 \%$ & $2.08 \%$ & $13.92 \%$ & $13.33 \%$ \\
\hline Life lesson/character development & $7.14 \%$ & $10.42 \%$ & $10.13 \%$ & $14.44 \%$ \\
\hline Personal accomplishment & $2.38 \%$ & $8.33 \%$ & $13.92 \%$ & $10.00 \%$ \\
\hline Long trip or vacation & $2.38 \%$ & $2.08 \%$ & $6.33 \%$ & $2.22 \%$ \\
\hline Celebration & $2.38 \%$ & $0.00 \%$ & $3.80 \%$ & $8.89 \%$ \\
\hline College transition & $0.00 \%$ & $10.42 \%$ & $2.53 \%$ & $3.33 \%$ \\
\hline
\end{tabular}


small number of expected events and memories rated negative.

Unexpected events Rubin et al. (2009) stated that "highly negative events typically consist of either deviations from the timing and sequencing of the life script or of nonscripted events" (p. 57). Accordingly, a substantial proportion of highly unexpected predicted events and memories should be rated negative, and temporal distributions of these negative memories should not show a bump. For unexpected predicted events and memories that are rated positive, two outcomes are possible. If temporal distributions do not show increases during the bump period, this would suggest that a search for positive or expected events, rather than positive emotions per se, is necessary to produce the bump. If temporal distributions of unexpected positive predicted events and memories do show a bump, this would pose a challenge to the idea that the bump is dependent on a search targeting scripted or normative life events. Discovering reminiscence bumps for memories of unexpected events would, at the very least, require a modification or novel application of the life script hypothesis.

\section{Study 3}

We asked college students to imagine a hypothetical 80year-old and to describe one highly expected and one highly unexpected event that this person would recall from across their life span. After participants described each event, they were asked to rate both the positive and negative valence of the event and to provide the age at which the hypothetical 80-year-old experienced the event.

\section{Method}

Questionnaire The questionnaire and procedure of Study 3 were identical to those in Study 1, with the following exceptions: Participants were asked to describe, in detail, both a highly expected and a highly unexpected event (counterbalanced), to rate both the positive (scale ranging from 1 to 5; $1=$ not at all positive, $5=$ extremely positive) and negative (scale ranging from 1 to $5 ; 1=$ not at all negative, 5 = extremely negative) valence of each event, and to rate how surprised the hypothetical 80-year-old would have been by the event (scale ranging from 1 to $5 ; 1=$ not at all surprised, 5 = extremely surprised).

Participants Of the 199 college students who completed the questionnaire, 3 participants were dropped for failure to follow instructions $(n=196)$. The majority $(87.2 \%)$ of participants self-identified as European, Caucasian, or White, females made up $73.5 \%(n=144)$ of the sample, and the mean age of participants was 18.59 years $(S D=$ 0.99 ; range $=17-26$ ).

Content coding The first author and a research assistant read all event descriptions, generated content categories for highly expected and highly unexpected predicted events, and coded all event descriptions. Categories capturing 3.0\% of events within expectedness were retained-all other events were folded into an "other" category-and differences were resolved through discussion. A second research assistant, blind to the hypotheses, coded a random sample of $30.0 \%$ of predicted events, resulting in $100.00 \%$ agreement $(\kappa=1.00)$ for highly expected events and $93.22 \%$ agreement $(\kappa=.92)$ for highly unexpected events.

\section{Results}

Highly unexpected events were rated as more surprising $(M=4.27, S D=0.99)$ than highly expected events $(M=2.16$, $S D=1.17), t(195)=19.49, p<.001, \eta^{2}=.66$.

Using a procedure described by Talarico et al. (2004; Rubin \& Talarico, 2009), a valence score was calculated for each predicted event on the basis of corresponding positive and negative ratings, using the formula [positive rating $+(6$ - negative rating $)] / 2=$ valence, such that a score of three was coded neutral, above three positive, and below three negative. As was predicted, a majority of highly expected events were coded positive $(81.63 \%$; negative $=$ $14.29 \%$, neutral $=4.08 \%$ ) and a majority of highly unexpected events were coded negative (77.04\%; positive $=20.41 \%$, neutral $=2.55 \%$ ).

Distributions of highly expected and unexpected predicted events are presented in Fig. 3. A large majority $(81.12 \%)$ of highly expected events were predicted to occur in the bump period, $\chi^{2}(3)=349.87, p<.001$, and nearly half $(44.39 \%)$ of highly unexpected events were predicted to occur in the bump period, $\chi^{2}(3)=72.63, p<.001$. Distributions of expected positive, expected negative, unexpected positive, and unexpected negative predicted events are presented in Fig. 4. For events rated positive, a sharp reminiscence bump is evident for both expected events $\left(90.63 \%\right.$ fall within the bump period), $\chi^{2}(3)=$ $384.41, p<.001$, and unexpected events $(80.00 \%$ fall within the bump period), $\chi^{2}(3)=69.00, p<.001$. For events rated negative, a smaller bump is evident for unexpected events $(35.76 \%$ fall within the bump period), $\chi^{2}(3)=30.06, p<.001$, and the distribution of expected events does not differ significantly from an equal distribution, $\chi^{2}(3)=4.29, p=.232$. 


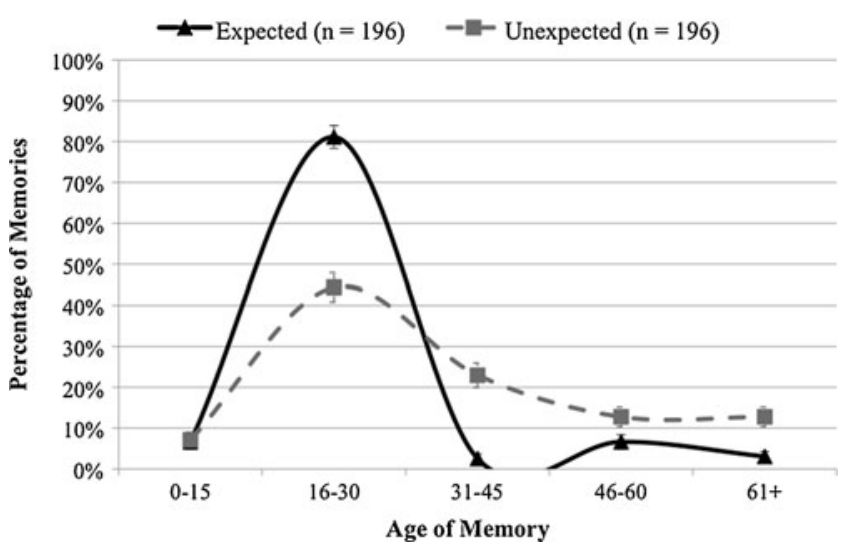

Fig. 3 Age distributions of expected and unexpected events predicted by college students (Study 3)

Table 4 (expected) and Table 5 (unexpected) present predicted content categories by bump period and valence. The vast majority of both positive expected and unexpected bump period events refer to one of two age-linked content categories: proposal or marriage and pregnancy or having a child. For negative unexpected bump period events, only pregnancy or having a child is strongly overrepresented relative to the nonbump period.

\section{Discussion}

Consistent with the life script hypothesis, but not previously reported in earlier studies, highly expected predicted events were predominately positive and highly unexpected predicted events were predominantly negative. Second, a large majority of positive expected events were predicted to occur in the bump period. Third, positive expected bump period events frequently reflected age-linked cultural landmarks.

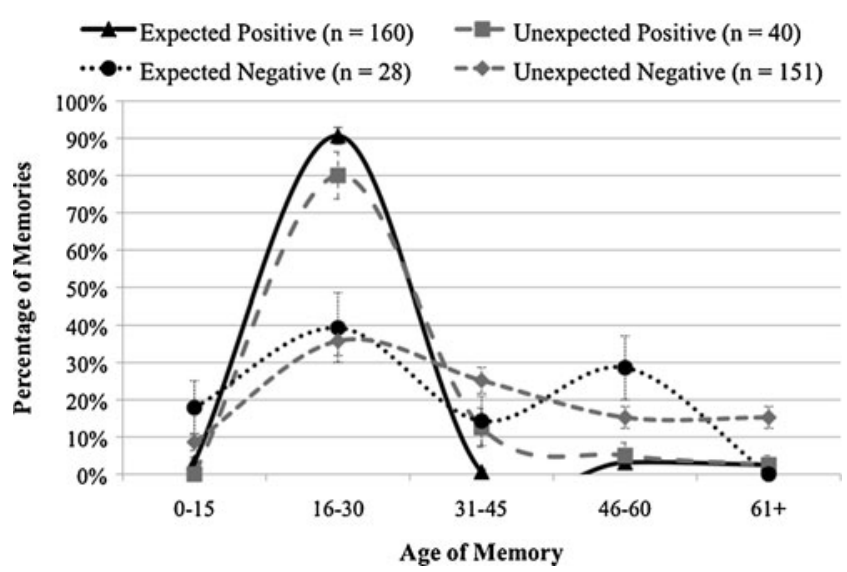

Fig. 4 Age distributions of expected positive, expected negative, unexpected positive, and unexpected negative events predicted by college students (Study 3)
The overall distribution of predicted unexpected events also showed a prominent reminiscence bump, albeit a smaller one than for predicted expected events. Notably, unexpected events rated positive showed a bump comparable to the bump for positive expected events, indicating that neither an explicit positive emotion cue nor a request for an expected event is necessary to direct students' predictions to late adolescence and early adulthood. In addition, the distribution of negative unexpected events showed a smaller but statistically significant bump. Study 4 tested whether these new findings based on students' predictions are apparent in actual memories reported by older adults.

\section{Study 4}

Adults were asked to provide detailed accounts of their own highly expected and highly unexpected memories from across the life span and to rate each memory on positive and negative valence only after providing their memory description.

\section{Method}

Questionnaire The design and method of Study 4 were identical to the design and method of Study 2, with the following exceptions: Older adults were asked to describe, in detail, both a specific personal memory of an event that was highly expected and a specific personal memory of an event that was highly unexpected (counterbalanced), to rate both the positive and negative valence of each memory, and to rate how surprised they were by the event (5-point scale).

Participants Older adults who had taken part in New Hampshire statewide political opinion polling from 2006 to 2008 (contact established using a random-digit dialing procedure) and who had agreed to be recontacted for research purposes were contacted by the University of New Hampshire (UNH) Survey Center to take part in the present study. In total, 1,430 adults age 50 and older were contacted about participation, and of those who agreed to receive our research materials, 888 possessed complete mailing addresses and were mailed a recruitment letter and questionnaire. Adults who agreed to participate were asked to complete the questionnaire and return it to UNH via an enclosed prepaid envelope. Compensation was provided in the form of two participant raffles, each for a $\$ 100$ gift certificate; raffle forms were returned separate from questionnaires. Reminder letters and debriefing letters were mailed to all the adults who had initially agreed to receive our research materials. 
Table 4 Study 3 expected content categories by bump period and valence rating

\begin{tabular}{|c|c|c|c|c|}
\hline \multirow[t]{2}{*}{ Category } & \multicolumn{2}{|c|}{ Bump Period (16-30 Years) } & \multicolumn{2}{|c|}{ Nonbump Period } \\
\hline & Positive & Negative & Positive & Negative \\
\hline$n$ & 145 & 11 & 15 & 17 \\
\hline Other & $7.59 \%$ & $27.27 \%$ & $40.00 \%$ & $58.82 \%$ \\
\hline Proposal or marriage & $65.52 \%$ & $18.18 \%$ & $6.67 \%$ & $0.00 \%$ \\
\hline Pregnancy or having child & $16.55 \%$ & $9.09 \%$ & $0.00 \%$ & $0.00 \%$ \\
\hline High school or college transition & $8.97 \%$ & $9.09 \%$ & $0.00 \%$ & $0.00 \%$ \\
\hline World War II related & $1.38 \%$ & $27.27 \%$ & $0.00 \%$ & $0.00 \%$ \\
\hline Death & $0.00 \%$ & $9.09 \%$ & $0.00 \%$ & $41.18 \%$ \\
\hline Grandchild & $0.00 \%$ & $0.00 \%$ & $53.33 \%$ & $0.00 \%$ \\
\hline
\end{tabular}

Of the 888 questionnaires mailed, 11 adults contacted us to decline participation, and 1 questionnaire was returned due to an incorrect address. Excluding these 12 adults, 398 questionnaires were returned at least partially completed (response rate $=45.43 \%$ ); 17 participants were dropped for failure to follow instructions, resulting in a sample of 381 participants. A majority of respondents were age 60 or older $(n=227)$. We limited our analyses to these older participants for several reasons. First, sampling adults age 60 and older is consistent with Study 2 sample characteristics and permits cross-study comparisons. Second, temporal distributions of memories for adults over age 60 can be displayed in four 15-year age bins, including the theoretically relevant 16 - to 30 -year bump interval; inclusion of adults below age 60 requires a different analytic strategy. Third, memory distributions for participants age 50-59, using five 10-year age bins, are consistent with the overall pattern of results for adults age 60 and older, although the smaller number of participants and the larger number of age bins produces more variability. For these reasons, all reported analyses are based on a final sample of 227 adults over age 60 .

Almost all (97.8\%) participants self-identified as European, Caucasian, or White, females made up $44.9 \%$ ( $n=$ 102) of the sample, and the mean age of participants was 68.65 years $(S D=6.81$; range $=60-89)$.

Content coding Using the coding scheme generated from Study 3 college student predictions, the first author and a research assistant coded all memories independently, and differences were resolved through discussion. A research assistant, blind to the hypotheses, then coded a random sample of $30.0 \%$ of the memories using the scheme generated from college student predictions, resulting in $92.96 \%$ agreement $(\kappa=.90)$ for highly expected memories and $87.32 \%$ agreement $(\kappa=.84)$ for highly unexpected memories. The coding scheme generated from college student predictions captured approximately half of the highly expected memories (46.70\% coded "other"; $33.92 \%$ of the highly unexpected memories coded "other").
Table 5 Study 3 unexpected content categories by bump period and valence rating

\begin{tabular}{|c|c|c|c|c|}
\hline \multirow[t]{2}{*}{ Category } & \multicolumn{2}{|c|}{ Bump Period (16-30 Years) } & \multicolumn{2}{|c|}{ Nonbump Period } \\
\hline & Positive & Negative & Positive & Negative \\
\hline$n$ & 32 & 54 & 8 & 97 \\
\hline Other & $21.88 \%$ & $14.81 \%$ & $50.00 \%$ & $9.28 \%$ \\
\hline Pregnancy or having child & $34.38 \%$ & $18.52 \%$ & $0.00 \%$ & $0.00 \%$ \\
\hline Proposal or marriage & $34.38 \%$ & $0.00 \%$ & $12.50 \%$ & $3.09 \%$ \\
\hline Career or finance related & $9.38 \%$ & $0.00 \%$ & $37.50 \%$ & $7.22 \%$ \\
\hline Accident or disease & $0.00 \%$ & $20.37 \%$ & $0.00 \%$ & $28.87 \%$ \\
\hline Other death & $0.00 \%$ & $14.81 \%$ & $0.00 \%$ & $10.31 \%$ \\
\hline Parent death & $0.00 \%$ & $12.96 \%$ & $0.00 \%$ & $6.19 \%$ \\
\hline Child death & $0.00 \%$ & $7.41 \%$ & $0.00 \%$ & $14.43 \%$ \\
\hline World War II related & $0.00 \%$ & $7.41 \%$ & $0.00 \%$ & $4.12 \%$ \\
\hline JFK assassination & $0.00 \%$ & $3.70 \%$ & $0.00 \%$ & $3.09 \%$ \\
\hline Spouse death & $0.00 \%$ & $0.00 \%$ & $0.00 \%$ & $13.40 \%$ \\
\hline
\end{tabular}


The two researchers next coded memories using additional categories generated from actual adult memory content, as well as categories reported by Berntsen and Rubin (2004), and differences were again resolved through discussion. The research assistant then coded a random sample of approximately $30.0 \%$ of the highly expected and highly unexpected memories originally coded "other," resulting in $78.79 \%$ agreement $(\kappa=.75)$ for highly expected memories and $80.95 \%$ agreement $(\kappa=.73)$ for highly unexpected memories.

\section{Results}

Highly unexpected memories $(M=4.49, S D=0.79)$ were rated as more surprising than highly expected memories $(M=2.37, S D=1.45), t(226)=20.36, p<.001, \eta^{2}=.65$.

Analysis of valence ratings (see Study 3 for the procedure used to calculate ratings) revealed that the majority of highly expected memories were coded positive $(79.30 \%$; negative $=16.74 \%$, neutral $=3.96 \%)$ and the majority of highly unexpected memories were coded negative $(64.32 \%$; positive $=30.84 \%$, neutral $=4.85 \%)$.

Distributions of highly expected and highly unexpected memories are presented in Fig. 5. A strong reminiscence bump is evident both for expected memories $(49.78 \%$ fall within the bump period), $\chi^{2}(3)=107.40, p<.001$, and for unexpected memories $(38.77 \%$ fall within the bump period), $\chi^{2}(3)=33.50, p<.001$. Distributions of expected positive, expected negative, unexpected positive, and unexpected negative memories are presented in Fig. 6. For memories rated positive, the distribution of expected memories shows a strong reminiscence bump $(53.89 \%$ of memories fall within the bump period), $\chi^{2}(3)=109.65, p<$ .001 , and the distribution of unexpected memories shows a strikingly similar reminiscence bump $(50.00 \%$ of memories

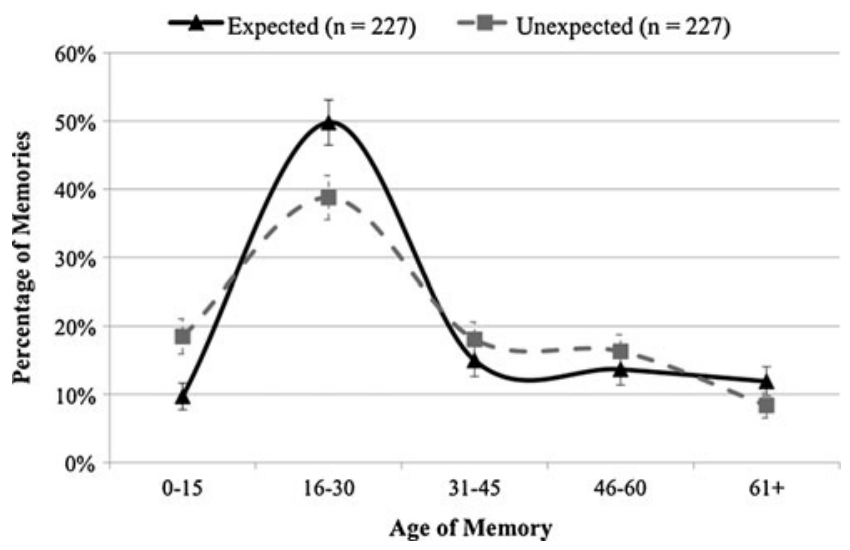

Fig. 5 Age distributions of expected and unexpected memories for older adults (Study 4)

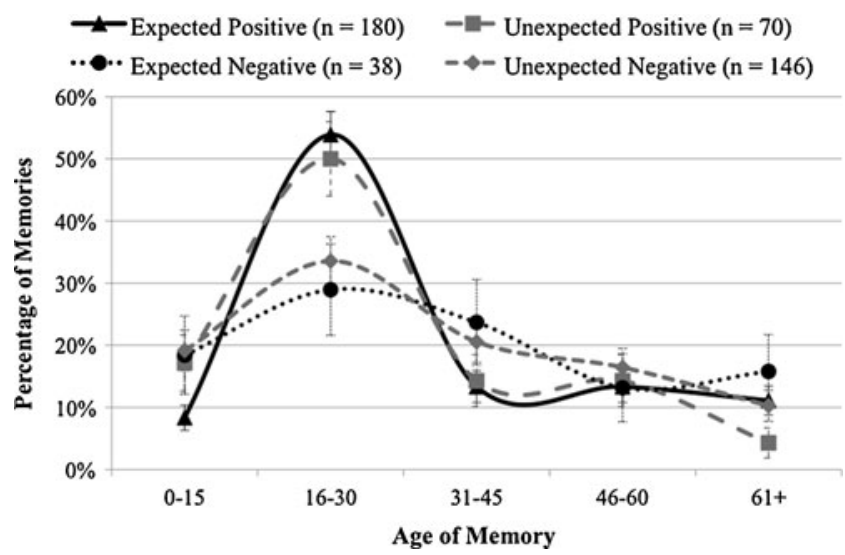

Fig. 6 Age distributions of expected positive, expected negative, unexpected positive, and unexpected negative memories for older adults (Study 4)

fall within the bump period), $\chi^{2}(3)=26.67, p<.001$. For memories rated negative, the distribution of unexpected memories shows a small but clearly discernible bump (33.56\% of memories fall within the bump period), $\chi^{2}(3)=$ $11.32, p=.010$, whereas the distribution of expected memories is relatively flat, $\chi^{2}(3)=2.50, p=.475$.

Table 6 presents content categories for highly expected memories by bump period and valence. For positive expected bump period memories, the categories of proposal or marriage, pregnancy or having a child, and high school or college transition are overrepresented, as compared with nonbump period memories.

Table 7 presents content categories for highly unexpected memories by bump period and valence. For positive unexpected bump period memories, "other," proposal or marriage, and pregnancy or having a child are overrepresented, as compared with nonbump period memories. For negative unexpected bump period memories, only the "other" category is strongly overrepresented as compared with nonbump period memories.

\section{Discussion}

Consistent with the life script hypothesis and with Study 3 student predictions, memories of highly expected events were predominately positive, and memories of highly unexpected events were predominantly negative. Also consistent with life script predictions, memories of expected events were overrepresented in the bump period, expected bump period memories frequently referenced age-linked scripted events, and the bump for highly expected events was found for positive, but not for negative, memories.

In agreement with Study 3 student predictions, the overall distribution of memories for highly unexpected events showed a clear reminiscence bump. As in Study 3, 
Table 6 Study 4 expected content categories by bump period and valence rating

Note. Study 3 predicted categories not reaching the 3.0\% criteria were folded into the category "other"

\begin{tabular}{|c|c|c|c|c|}
\hline \multirow[t]{2}{*}{ Category } & \multicolumn{2}{|c|}{ Bump Period (16-30 Years) } & \multicolumn{2}{|c|}{ Nonbump Period } \\
\hline & Positive & Negative & Positive & Negative \\
\hline$n$ & 97 & 11 & 83 & 27 \\
\hline Other & $9.28 \%$ & $27.27 \%$ & $26.51 \%$ & $14.81 \%$ \\
\hline Proposal or marriage & $26.80 \%$ & $0.00 \%$ & $10.84 \%$ & $14.81 \%$ \\
\hline Pregnancy or having child & $24.74 \%$ & $9.09 \%$ & $12.05 \%$ & $0.00 \%$ \\
\hline High school or college transition & $11.34 \%$ & $27.27 \%$ & $4.82 \%$ & $0.00 \%$ \\
\hline Accomplishment/disappointment & $11.34 \%$ & $9.09 \%$ & $10.84 \%$ & $3.70 \%$ \\
\hline Career or finance related & $11.34 \%$ & $0.00 \%$ & $13.25 \%$ & $7.41 \%$ \\
\hline Long trip or vacation & $4.12 \%$ & $0.00 \%$ & $8.43 \%$ & $0.00 \%$ \\
\hline Death & $1.03 \%$ & $9.09 \%$ & $3.61 \%$ & $40.74 \%$ \\
\hline Accident or disease & $0.00 \%$ & $18.18 \%$ & $2.41 \%$ & $11.11 \%$ \\
\hline Celebration or rite & $0.00 \%$ & $0.00 \%$ & $7.23 \%$ & $7.41 \%$ \\
\hline
\end{tabular}

the bump was prominent for unexpected positive memories and, to a lesser extent, for unexpected negative memories. The bump for positive unexpected memories contained an overrepresentation of events categorized as "other," as well as events usually characterized as normative: pregnancy or having a child and proposal or marriage. The bump for negative unexpected events contained an overrepresentation only of events falling into the "other" category. The presence of reminiscence bumps for unexpected positive and negative memories requires, at the very least, a theoretical expansion or modification of the life script hypothesis.

\section{General discussion}

According to Rubin and Berntsen (2003; Berntsen \& Rubin, 2002, 2004), individuals within a given culture possess knowledge as to the type and timing of important landmark events that one is likely to experience over the course of his or her life-a life script. Because negative events are often unpredictable or, when predictable, are not linked to a narrow age range, the life script is composed primarily of positive events. When older adults are asked to recall emotionally positive memories, the script guides the memory search to late adolescence and early adulthoodthe life period overpopulated with positive scripted events. Empirical support for the life script hypothesis is extensive. When college students predict events likely to be recounted by a prototypical older adult, or when adults recount their own salient emotional memories, a bump is evident for positive, but not negative events (e.g., Berntsen \& Rubin, 2004). In addition, content analyses of positive bump period memories identify a high incidence of scripted transitional events (e.g., Thomsen \& Berntsen, 2008).

Although prior studies are consistent with the idea that a cultural life script guides the retrieval of positive memories, the supporting evidence is indirect. Positive memories of
Table 7 Study 4 unexpected content categories by bump period and valence rating

Note. Because the Study 3 predicted categories "spouse death" and "child death" did not reach the $3.0 \%$ criteria, they were combined with the category "other death." All other predicted categories not reaching $3.0 \%$ were folded into the category "other"

\begin{tabular}{|c|c|c|c|c|}
\hline \multirow[t]{2}{*}{ Category } & \multicolumn{2}{|c|}{ Bump Period (16-30 Years) } & \multicolumn{2}{|c|}{ Nonbump Period } \\
\hline & Positive & Negative & Positive & Negative \\
\hline$n$ & 35 & 49 & 35 & 97 \\
\hline Other & $31.43 \%$ & $26.53 \%$ & $20.00 \%$ & $12.37 \%$ \\
\hline Career or finance related & $22.86 \%$ & $12.24 \%$ & $20.00 \%$ & $8.25 \%$ \\
\hline Proposal or marriage & $14.29 \%$ & $8.16 \%$ & $5.71 \%$ & $12.37 \%$ \\
\hline Pregnancy or having child & $11.43 \%$ & $0.00 \%$ & $0.00 \%$ & $1.03 \%$ \\
\hline Nonromantic relationship & $8.57 \%$ & $2.04 \%$ & $17.14 \%$ & $3.09 \%$ \\
\hline Accomplishment/disappointment & $5.71 \%$ & $2.04 \%$ & $11.43 \%$ & $1.03 \%$ \\
\hline Accident or disease & $2.86 \%$ & $10.20 \%$ & $22.86 \%$ & $27.84 \%$ \\
\hline Life lesson/character development & $2.86 \%$ & $4.08 \%$ & $2.86 \%$ & $1.03 \%$ \\
\hline Other death & $0.00 \%$ & $14.29 \%$ & $0.00 \%$ & $15.46 \%$ \\
\hline Parent death & $0.00 \%$ & $12.24 \%$ & $0.00 \%$ & $11.34 \%$ \\
\hline Victimization or discrimination & $0.00 \%$ & $8.16 \%$ & $0.00 \%$ & $6.19 \%$ \\
\hline
\end{tabular}


scripted events could be overrepresented in late adolescence and early adulthood without requiring that a life script actively direct the retrieval process. For example, events that achieve cultural landmark status, such as childbirth or marriage, could elicit high emotions and receive preferential attention and rehearsal at the time of encoding. The search for memories years later could be driven by subjectively experienced emotional salience and distinctiveness, rather than by close adherence to a higher order script.

To clarify the role played by a life script in producing the reminiscence bump, we examined memories of standard, surprising, expected, and unexpected events. In Study 1, college students' predictions for especially positive and negative events were consistent with prior research and the life script hypothesis: A bump was evident for positive, but not negative, events, and positive bump period event descriptions predominantly referred to scripted transitions or landmarks. In Study 2, older adults' memories showed a parallel bump for positive memories only, and references to major cultural landmarks - notably, pregnancy or having a child and proposal or marriage-were overrepresented in the bump period.

The distributions of surprising positive predicted events and memories also demonstrated prominent reminiscence bumps, and content analyses revealed an overrepresentation of landmark events (e.g., pregnancy or having a child, college transition) in the bump compared to nonbump periods. These results are also consistent with the life script perspective: A positive emotion cue first guides the memory search to the period of life overrepresented by positive events (Berntsen \& Rubin, 2004), from which an individual then searches for an event with a surprising element.

Prior studies have indicated that the word surprise has a slightly positive connotation (e.g., Bradley \& Lang, 1999). Studies 3 and 4 more clearly separated expectedness from emotional valence by asking college students to predict and older adults to recall highly expected and highly unexpected events and memories. Participants rated valence only after providing event descriptions. Two key life script predictions - that the majority of highly expected events and memories would be rated positive and that a bump would be evident for expected positive events and memories only-were confirmed. Also consistent with life script predictions, content categories reflecting cultural landmarks were overrepresented in highly expected bump period events and memories.

In contrast, our discovery of reminiscence bumps for unexpected predicted events and actual memories raises new questions for the life script hypothesis. When predicted events and memories were separated on the basis of emotion ratings, distributions of unexpected positive events and memories showed classic reminiscence bumps, almost identical in magnitude to the bumps for expected positive events and memories. In addition, clearly discernable bumps were evident even for unexpected negative predicted events and actual memories.

Content analyses of unexpected memories suggest that nonscripted events frequently populate the bump period. For unexpected negative memories, "other" is the only category overrepresented in the bump period. Notably, pregnancy or having a child, the one content category that was prominently overrepresented in the bump for students' predictions of unexpected negative events, is missing entirely from adults' actual bump period memories. For unexpected positive bump period memories, "other" is the most frequent content category, and it is more prevalent in the bump than in the nonbump period. The categories proposal or marriage and pregnancy or having a child are also overrepresented in the bump period for unexpected positive memories, even though marriage and having children are normative scripted events (Berntsen \& Rubin, 2004).

In summary, when memories are elicited with an explicit positive or negative emotion cue, our findings for both standard and surprising events are consistent with the life script hypothesis. A request for expected events absent an emotion cue also produced the predicted pattern of results. In contrast, finding a reminiscence bump for memories of unexpected events poses a theoretical challenge. Can the life script hypothesis be adapted to account for these findings, or are other explanations necessary?

Support for the life script account comes from the highly similar temporal distributions of students' predicted events and adults' actual memories for all prompts, including the request for unexpected events. Students were able to anticipate not only the prominent bump for positive unexpected events, but also the smaller bump for negative unexpected events, suggesting a shared cultural understanding of when unexpected events are likely to occur. Students also accurately predicted the overrepresentation in adults' unexpected positive bump period memories of proposal or marriage and pregnancy or having a child, although the most frequent adult memory category was "other." In contrast, the category pregnancy or having a child, prominent in students' predictions of negative unexpected bump period events, was missing from adults' actual memories; the increased frequency of bump period, as compared with nonbump period, memories was attributable primarily to an overrepresentation of memories coded as "other." Life script theory could potentially account for these findings if the script contains prescriptions for the timing of unexpected, as well as expected, events, but not necessarily their specific content. That is, shared cultural knowledge could locate unexpected positive events and, to a lesser extent, negative events in late adolescence and early adulthood and direct the memory search to this life period. 
But if the cultural script directs the search for both expected and unexpected events to the same life period, its unique predictive power is diminished.

Another possibility is that the request for a memory of an unexpected event activates, rather than bypasses, the life script for expected events. When people are asked to recall highly unexpected events, they may think first of highly expected events and then search for script violations, when things did not go entirely as expected. Although events like marriage and childbirth are part of the general life script, they may also contain unanticipated elements. A memory search targeting variations from the script could account for the overrepresentation of unexpected positive bump period memories relating to pregnancy or having a child and proposal or marriage. Nevertheless, a majority of unexpected memories focused on negative outcomes - events that are not part of the life script. It is unclear why a life script composed primarily of positive normative events would be activated by a request for unexpected events only for a minority of participants. In addition, the life script hypothesis cannot readily account for the overrepresentation of unexpected bump period memories categorized as "other."

A complete explanation for the reminiscence bump may require a combination of theoretical perspectives. For example, proponents of a personal identity or selfnarrative perspective emphasize the persistence and heightened accessibility of memories relevant to one's sense of self and development during the critical transitional period of late adolescence and early adulthood (e.g., Conway, 2005; Fitzgerald, 1999; Glück \& Bluck, 2007). Unexpected positive and negative events populating the bump period could play a critical role in an individualized self-narrative, including the common but idiosyncratic episodes categorized as "other."

Another explanation builds on the straightforward idea that some events of early adolescence and young adulthood remain highly accessible years after their occurrence because they possess fundamental qualities known to enhance memory vividness (e.g., Rubin \& Kozin, 1984): They are subjectively experienced as highly emotional, important, and distinctive, and as a result, they are frequently rehearsed. The scripted positive landmark events of late adolescence and early adulthood are likely to be accompanied by high emotions, to capture focused attention, and to be rehearsed regularly in the years that follow. Marriage, childbirth, and the transition to college are highly distinctive and eagerly anticipated; they profoundly affect the life trajectory, and as such, they are frequently thought about and talked about. When a memory probe instead targets unexpected occurrences, some events overrepresented in the bump period may share qualities such as emotionality, distinctiveness, personal importance, and rehearsal frequency without requiring an activated cultural life script to guide their retrieval.

In conclusion, our results provide additional support for the life script hypothesis but also raise new questions. Future research is needed to clarify the precise role played by an activated life script during recall of salient personal events. Think-aloud protocols, in which older adults describe their ongoing thoughts as they search for memories, may clarify the cognitive processes contributing to the reminiscence bump. In addition, memory content and temporal distributions could be compared for people who differ in the extent to which their individualized self-narratives conform to the culturally shared life script. For example, in the present studies, overrepresented positive predicted events and actual memories focused primarily on two content categories: proposal or marriage and pregnancy or having a child. Temporal distributions of memories for people who are single and childless could be compared with memories of people who are married with children to determine whether the bump depends on actual experiences in particular content domains. Other studies could selectively activate or prime the life script separately from the recall task and then observe its impact on memory. New studies will help to determine whether a shared cultural life script is necessary to direct the memory search to early adolescence and young adulthood, or whether a bump is evident even in its absence, because the remembered events represent significant and emotional episodes in personal life narratives.

\section{References}

Berntsen, D., \& Rubin, D. C. (2002). Emotionally charged autobiographical memories across the life-span: The recall of happy, sad, traumatic and involuntary memories. Psychology and Aging, 17, 636-652. doi:10.1037//0882-7974.17.4.636

Berntsen, D., \& Rubin, D. C. (2004). Cultural life scripts structure recall from autobiographical memory. Memory \& Cognition, 32, 427-442.

Berntsen, D., \& Rubin, D. C. (2006). The centrality of event scale: A measure of integrating a trauma into one's identity and its relation to post-traumatic stress disorder symptoms. Behaviour Research and Therapy, 44, 219-231. doi:10.1016/j.brat.2005.01.009

Bluck, S., \& Habermas, T. (2000). The life story schema. Motivation and Emotion, 24, 121-145. doi:10.1023/A:1005615331901

Bluck, S., \& Habermas, T. (2001). Extending the study of autobiographical memory: Thinking back across the life span. Review of General Psychology, 5, 135-147. doi:10.1037/1089-2680.5.2.135

Bohn, A. (2009). Generational differences in cultural life scripts and life story memories of younger and older adults. Applied Cognitive Psychology, 24, 1324-1345. doi:10.1002/acp.1641

Bohn, A., \& Berntsen, D. (2008). Life story development in children: The development of life story abilities and the acquisition of cultural life scripts from late middle childhood to adolescence. Developmental Psychology, 44, 1135-1147. doi:10.1037/0012-1649.44.4.1135

Bradley, M. M., \& Lang, P. J. (1999). Affective norms for English words (ANEW): Instruction manual and affective ratings (Tech. Rep. C-1). Gainesville: University of Florida, Center for Research in Psychophysiology. 
Chu, S., \& Downes, J. J. (2000). Long live Proust: The odour-cued autobiographical memory bump. Cognition, 75, B41-B50. doi:10.1016/S0010-0277(00)00065-2

Collins, K. A., Pillemer, D. B., Ivcevic, Z., \& Gooze, R. A. (2007). Cultural life scripts guide recall of intensely positive life events. Memory \& Cognition, 35, 651-659.

Conway, M. A. (2005). Memory and the self. Journal of Memory and Language, 53, 594-628. doi:10.1016/j.jml.2005.08.005

Conway, M. A., \& Haque, S. (1999). Overshadowing the reminiscence bump: Memories of a struggle for independence. Journal of Adult Development, 6, 35-44. doi:10.1023/A:1021672208155

Conway, M. A., \& Holmes, A. (2004). Psychosocial stages and the accessibility of autobiographical memories across the life cycle. Journal of Personality, 72, 461-480. doi:10.1111/ j.0022-3506.2004.00269.x

Conway, M. A., Wang, Q., Hanyu, K., \& Haque, S. (2005). A crosscultural investigation of autobiographical memory: On the universality and cultural variation of the reminiscence bump. Journal of Cross-Cultural Psychology, 36, 739-749. doi:10.1177/ 0022022105280512

Davison, I. M., \& Feeney, A. (2008). Regret as autobiographical memory. Cognitive Psychology, 57, 385-403. doi:10.1016/j. cogpsych.2008.03.001

Elnick, A. B., Margrett, J. A., Fitzgerald, J. M., \& Labouvie-Vief, G. (1999). Benchmark memories in adulthood: Central domains and predictors of their frequency. Journal of Adult Development, 6, 45-59. doi:10.1023/A:1021624324994

Erdoğan, A., Baran, B., Avlar, B., Çağlar Taş, A., \& Tekcan, A. İ. (2008). On the persistence of positive events in life scripts. Applied Cognitive Psychology, 22, 95-111. doi:10.1002/acp.1363

Fitzgerald, J. M. (1988). Vivid memories and the reminiscence phenomenon: The role of a self narrative. Human Development, 31, 261-273. doi:10.1159/000275814

Fitzgerald, J. M. (1996). The distribution of self-narrative memories in younger and older adults: Elaborating the self-narrative hypothesis. Aging, Neuropsychology, and Cognition, 3, 229-236. doi:10.1080/ 13825589608256626

Fitzgerald, J. M. (1999). Autobiographical memory and social cognition: Development of the remembered self in adulthood. In T. M. Hess \& F. Blanchard-Fields (Eds.), Social cognition and aging (pp. 143-171). New York: Academic Press. doi:10.1016/ B978-012345260-3/50008-2

Glück, J., \& Bluck, S. (2007). Looking back across the life span: A life story account of the reminiscence bump. Memory \& Cognition, 35, 1928-1939.

Habermas, T. (2007). How to tell a life: The development of the cultural concept of biography. Journal of Cognition and Development, 8, 1-31. doi:10.1207/s15327647jcd0801_1

Holmes, A., \& Conway, M. A. (1999). Generation identity and the reminiscence bump: Memory for public and private events. Journal of Adult Development, 6, 21-34. doi:10.1023/A:1021620224085

Jansari, A., \& Parkin, A. J. (1996). Things that go bump in your life: Explaining the reminiscence bump in autobiographical memory. Psychology and Aging, 11, 85-91. doi:10.1037/0882-7974.11.1.85

Janssen, S. M. J., Chessa, A. G., \& Murre, J. M. J. (2005). The reminiscence bump in autobiographical memory: Effects of age, gender, education, and culture. Memory, 13, 658-668. doi:10.1080/ 09658210444000322

Janssen, S. M. J., Murre, J. M. J., \& Meeter, M. (2008). Reminiscence bump in memory for public events. European Journal of Cognitive Psychology, 20, 738-764. doi:10.1080/09541440701554409

Janssen, S. M. J., \& Rubin, D. C. (2011). Age effects in cultural life scripts. Applied Cognitive Psychology. doi:10.1002/acp.1690

Robinson, J., \& Taylor, L. (1998). Autobiographical memory and selfnarratives: A tale of two stories. In C. P. Thompson, D. J. Herrmann, D. Bruce, J. D. Read, D. G. Payne, \& M. P. Toglia
(Eds.), Autobiographical memory: Theoretical and applied perspectives (pp. 125-143). Mahwah: Erlbaum.

Rubin, D. C., \& Berntsen, D. (2003). Life scripts help to maintain autobiographical memories of highly positive, but not highly negative, events. Memory \& Cognition, 31, 1-14.

Rubin, D. C., Berntsen, D., \& Hutson, M. (2009). The normative and the personal life: Individual differences in life scripts and life story events among U.S.A. and Danish undergraduates. Memory, 17, 54-68. doi:10.1080/09658210802541442

Rubin, D. C., \& Kozin, M. (1984). Vivid memories. Cognition, 16, 81-95. doi:10.1016/0010-0277(84)90037-4

Rubin, D. C., Rahhal, T. A., \& Poon, L. W. (1998). Things learned in early adulthood are remembered best. Memory \& Cognition, 26, 3-19.

Rubin, D. C., \& Schulkind, M. D. (1997). The distribution of autobiographical memories across the lifespan. Memory \& Cognition, 25, 859-866.

Rubin, D. C., \& Talarico, J. M. (2009). A comparison of dimensional models of emotion: Evidence from emotions, prototypical events, autobiographical memories, and words. Memory, 17, 802-808. doi:10.1080/09658210903130764

Rubin, D. C., Wetzler, S. E., \& Nebes, R. D. (1986). Autobiographical memory across the lifespan. In D. C. Rubin (Ed.), Autobiographical memory (pp. 202-221). New York: Cambridge University Press.

Schrauf, R. W., \& Rubin, D. C. (1998). Bilingual autobiographical memory in older adult immigrants: A test of cognitive explanations of the reminiscence bump and the linguistic encoding of memories. Journal of Memory and Language, 39, 437-457. doi:10.1006/jmla.1998.2585

Schrauf, R. W., \& Rubin, D. C. (2001). Effects of voluntary immigration on the distribution of autobiographical memory over the lifespan. Applied Cognitive Psychology, 15, S75-S88. doi:10.1002/acp. 835

Schulkind, M. D., \& Woldorf, G. M. (2005). Emotional organization of autobiographical memory. Memory \& Cognition, 33, 1025-1035.

Talarico, J. M., LaBar, K. S., \& Rubin, D. C. (2004). Emotional intensity predicts autobiographical memory experience. Memory \& Cognition, 32, 1118-1132.

Thomsen, D. K., \& Berntsen, D. (2008). The cultural life script and life story chapters contribute to the reminiscence bump. Memory, 16, 420-435. doi:10.1080/09658210802010497

Waters, R. (1972). Free four [Recorded by Pink Floyd]. On Obscured by clouds [CD]. London: Harvest/EMI Records.

Webster, J. D., \& Gould, O. (2007). Reminiscence and vivid personal memories across adulthood. International Journal of Aging \& Human Development, 64, 149-170. doi:10.2190/Q8V4-X5H06457-5442

\section{Author Note}

The present research was conducted in partial fulfillment of requirements for the doctoral degree in the Department of Psychology at the University of New Hampshire for the first author and was supported, in part, by a Summer Teaching Assistant Fellowship and a Dissertation Year Fellowship awarded by the UNH Graduate School, as well as by the Dr. Samuel E. Paul Chair of Developmental Psychology held by David Pillemer. The authors would like to thank Kie Kuwabara, Anna Waller, Teresa Ware, Tanya Rouleau, Michelle Leichtman, David Rubin, Matthew Schulkind, Rebecca Warner, and Suzanne Graham for their invaluable assistance in the completion of this research, as well as Karl Pillemer, Myra Sabir, the Cornell Institute for Translational Research on Aging, and the UNH Survey Center for their assistance with participant recruitment and data collection. 